\title{
Recursos metodológicos para la adquisición de competencias y la inserción del alumnado con la realidad de las personas sin hogar (PSH).
}

\section{Methodological resources for the acquisition of competences and the students' insertion with regard to the reality of homeless people.}

\author{
Carmen García de Viguera ${ }^{1}$, Rafaela Mª Herrero Martínez², Salvador Ruiz \\ Pino $^{3} \&$ Jesús Poyato Varo 4
}

Fecha de recepción: 14/06/2018; Fecha de revisión: 27/06/2019; Fecha de aceptación: 05/07/2019

Cómo citar este artículo:

García, C., Herrero, R., Ruiz, S. \& Poyato, J. (2019). Recursos metodológicos para la adquisición de competencias y la inserción del alumnado con la realidad de las personas sin hogar (PSH). Revista de Innovación y Buenas Prácticas Docentes, 8(3), 43-54.

Autor de Correspondencia:fe1hemar@uco.es

\begin{abstract}
Resumen:
El proyecto de innovación docente presentado pretende concienciar y sensibilizar a los estudiantes de la realidad de las Personas Sin Hogar (PSH), para adquirir valores de respeto, solidaridad y empatía por este sector de la sociedad, a través de actividades académicamente dirigidas (ADD), empleando una metodología activa y participativa centrada en el trabajo grupal y cooperativo que facilite el logro de competencias propias de su titulación. Para ello, se planificaron una serie de actividades que favorecieron la coordinación horizontal y vertical de las materias participantes en las Titulaciones de Grado en Educación Infantil y de Grado en Educación Primaria del Centro de Magisterio "Sagrado Corazón".

De esta forma, los resultados implican la conexión y continuidad entre las materias participantes. Por último, las conclusiones muestran que los estudiantes universitarios interiorizan los valores necesarios para la transferencia de aprendizajes adquiridos, a través de la experiencia vivencial con la realidad de las PSH, la cual permite una mirada conjunta de la inclusión y la invisibilidad social, empleando un enfoque participativo donde las opiniones e inquietudes de ambos colectivos se tengan en cuenta. Para ello, es preciso no poner barreras excluyentes a la educación del estudiante como futuro docente.
\end{abstract}

Palabras clave: actividades, competencias, grupos cooperativos, personas sin hogar.

Abstract:

The present innovative teaching proyect aims at raising awareness and moving students about the reality of homeless people, in order to acquire values of respect, solidarity and empathy for this society sector, through

\footnotetext{
${ }^{1}$ Centro de Magisterio Sagrado Corazón, Universidad de Córdoba (España), z82gavim@uco.es; CÓDIGO ORCID: 0000-0002-7582-4165

2Centro de Magisterio Sagrado Corazón, Universidad de Córdoba (España), fe1hemar@uco.es; CÓDIGO ORCID: 0000-0002-5160-8206

${ }^{3}$ Centro de Magisterio Sagrado Corazón, Universidad de Córdoba (España), d02rupis@uco.es; CóDIGO ORCID: 0000-0003-4608-9314

${ }^{4}$ Centro de Magisterio Sagrado Corazón, Universidad de Córdoba (España), jpvaro@uco.es
} 
curricular activities, implementing an active and participatory methodology based on group and cooperative work which promotes the attainment of competences of their own degree. For this purpose, a set of activities that favoured horizontal and vertical coordination of the subjects involved in the Degrees in Early Childhood Education and Primary Education of the "Sagrado Corazón" Teacher Training School were planned.

Thus, the outcomes involve the connection and the continuity between the subjects concerned. Finally, the findings show that university students internalize the values necessary for the transfer of learning acquired, through the lived experience with the reality of homelessness, which allows for an overall perspective of inclusion and social invisibility, by applying a participatory approach where views and concerns of both sides are given due consideration. This requires the removal of exclusive barriers to the education of the student as a future teacher.

Key Words: activities, competences, cooperative groups, homeless people. 


\section{INTRODUCCIÓN}

Este proyecto de innovación se encuadra en la línea de acción prioritaria denominada Actividades Académicamente Dirigidas (AAD).

Los beneficiarios del mismo han sido los estudiantes de Grado en Educación Infantil y Grado en Educación Primaria, del Centro de Magisterio "Sagrado Corazón", para optimizar el rendimiento académico, profesional y personal de los discentes desde su formación inicial, enriqueciendo a su vez, su futura labor docente.

El plan de estudios del Espacio Europeo de Educación Superior (EEES) permite la innovación e investigación docente, en el ámbito universitario. Por ello, la enseñanza debe aunar competencias, objetivos y criterios, apreciando el trabajo grupal e interdisciplinar del alumnado. En consecuencia, esto obliga al profesorado participante a instruir a los estudiantes en el conocimiento desde una perspectiva diferente, que les forme como personas, adquiriendo valores que fomenten la tolerancia, la integración, la empatía y el respeto. A su vez, las competencias, habilidades y destrezas adquiridas por los discentes universitarios serán puestas en práctica en las aulas escolares.

Del mismo modo, clave para la formación del magisterio, es que los estudiantes desarrollen su creatividad e imaginación a través de la realización de recursos didácticos que faciliten la comprensión de los contenidos impartidos en las aulas y deberían "cumplir tres funciones: función motivadora, función de apoyo a la presentación del contenido y función estructuradora" (Rodríguez, 2005, p.57). De esta forma, las AAD, se complementan en su totalidad con el material didáctico elaborado, convirtiéndose en tareas de aprendizaje más exhaustivo y completo del alumnado centrado en el logro de competencias.

Por otro lado, para Palacios (2010), es muy importante entender el arte como comunitario, entendiendo este como una forma de involucrar a un grupo, asociación, etnia, etc., con el mundo artístico, de tal forma que colaborando conjuntamente se enriquezcan ambas partes del proceso y desarrollo creativo.

En este sentido, el origen del proyecto surge como resultado de la observación y reflexión conjunta de varios profesores del centro, sobre la situación que viven las Personas Sin Hogar (PSH) sumergidas en la exclusión y en la invisibilidad social.

A su vez, gracias al apoyo continuo e incondicional de la Casa de Acogida "Madre del Redentor" de Cáritas Diocesana, muchas de estas personas han conseguido salir o están en proceso de abandonar estas condiciones. Su experiencia y su lucha diaria por construirse un futuro digno, es lo que hace de ellas, que sean un ejemplo de superación y de fortaleza para los futuros docentes, en cuyas manos recae la educación del alumnado de la sociedad del mañana.

En esta línea, según Trilla (2007) el ambiente distendido entre los docentes y los discentes favorece las relaciones de confianza que propician la metodología de esta innovación ya que el alumnado obtiene una visión concreta de la realidad social que fomenta la solidaridad.

Por tanto, este proyecto de innovación educativa pretende que los estudiantes universitarios se conciencien de esta realidad. Así, para, Colom, Bernabeu, Domínguez \& Sarramona (2008), el proceso cognitivo, el discernimiento personal y la madurez moral del alumnado evolucionan al mismo tiempo que se desarrollan los valores éticos en los discentes, sin embargo, dicho proceso madurativo no responde a un acto espontáneo y aislado, sino que es consecuencia de las acciones sociales experimentadas por si mismos e interrelacionándose con los demás. 
Asimismo, para el desarrollo de dicho proyecto se utilizarán la representación escénica y teatral como vehículos para la transferencia del conocimiento a través de la experiencia vivencial de dicha realidad, y la transmisión del aprendizaje por medio del trabajo cooperativo, estableciendo de esta forma lazos de colaboración entre ambos ámbitos, social y educativo.

Las asignaturas implicadas en el proyecto son: Planificación e Innovación Educativa en Educación Primaria; Convivencia Escolar y Cultura de Paz en Educación Primaria; Educación Plástica y Visual, materias pertenecientes al título de Educación Primaria; Expresión Plástica Infantil y su Didáctica y Habilidades Comunicativas, Sociales, de Gestión y Emprendimiento para la Función Docente, materias pertenecientes al título de Educación Infantil.

Finalmente, con la planificación de $A A D$ se perseguirá una coordinación horizontal y vertical para el logro de las competencias de las materias participantes en las dos titulaciones en las que se desarrollará el proyecto de innovación educativa. Al mismo tiempo, segun Eisner (citado en Chisaguano, 2016), las sesiones plásticas y artísticas despiertan y potencian la mente en los discentes, construyendo personas creativas, con destreza expresiva y libertad emocional, reflexionando sobre si mismos para comprender a los demás.

\section{DESARROLLO DE LA EXPERIENCIA DE INNOVACIÓN}

\subsection{Objetivos}

La finalidad educativa del proyecto de innovación es concienciar y sensibilizar a los estudiantes de la realidad de las PSH, para adquirir valores de respeto, solidaridad y empatía por este sector de la sociedad, a través del trabajo grupal y cooperativo.

Para ello, se establecen los siguientes objetivos:

- Desarrollar competencias de las materias participantes en el proyecto de la titulación de Grado en Educación Infantil y Grado en Educación Primaria del Centro de Magisterio "Sagrado Corazón".

- Desempeñar metodologías innovadoras centradas en un método activo y participativo entre los discentes que cursan las materias implicadas.

- Mejorar la formación integral del alumnado universitario a través de actividades académicamente dirigidas.

\subsection{Material y métodos}

La metodología propuesta para las actividades programadas será activa y participativa, basada en el diálogo. La aplicación de esta metodología requiere utilizar equipos de trabajo para potenciar el aprendizaje cooperativo del estudiante, favoreciendo el logro de competencias a través de los talleres educativos.

La innovación implementada requiere la coordinación del profesorado participante puesto que las tareas diseñadas presentan un enfoque interdisciplinar. las siguientes:

El proyecto de innovación elaborado conlleva la aplicación de tres fases, que son

Fase I. Preparación: Información y Concienciación. Las actividades propuestas van dirigidas a las dos titulaciones participantes en el proyecto y serán, una charla informativa y una salida para vivenciar la problemática de las $\mathrm{PSH}$.

Respecto a la primera actividad, fue realizada por los responsables de Cáritas Diocesana, en el Centro de Magisterio "Sagrado Corazón" durante el mes de octubre para dar a conocer las funciones realizadas por la Casa de Acogida "Madre del 
Redentor" y concienciar al alumnado sobre la problemática social acontecida de este colectivo.

A su vez, la actividad de la salida fue asistir presencialmente a la Casa de Acogida para vivenciar in situ los procesos de gestión y coordinación de esta.

Fase II. Ejecución. Se han llevado a cabo distintos tipos de actividades basadas en el diseño y adaptación de materiales para la etapa evolutiva a la que van dirigidas; a su vez, el alumnado interactuará con las PSH mediante grupos de trabajo cooperativo. En este sentido, siguiendo a Arnheim citado en Chisaguano (2016) "la gran variedad de materiales artísticos, auxilian a que los niños, niñas y adolescentes despierten su creatividad; para luego lograr expresar nuevas y diferentes formas de invención". (p. 12).

Asimismo, para diferenciar la implemeantación de las actividades diseñadas, se presentarán por titulaciones.

En primer lugar, con el alumnado de Educación Primaria se diseñaron dos actividades: las vidrieras y la representación escénica.

Las materias participantes fueron las siguientes: Educación Plástica y Visual; Planificación e Innovación Educativa en Educación Primaria y Convivencia Escolar y Cultura de Paz en Educación Primaria.

Por otro lado, para dar respuesta a los objetivos planteados, fué necesario partir de las competencias comunes de las materias, para lo cual, se realizó una selección de las mismas y en base a ello se planificaron las tareas (véase figura 1).

\begin{tabular}{|c|c|}
\hline ASIGNATURAS & COMPETENCIAS \\
\hline Educación Plástica y Visual & $\begin{array}{l}\text { - CB2. Que los estudiantes sepan aplicar sus } \\
\text { conocimientos a su trabajo o vocación de una forma } \\
\text { profesional y posean las competencias que suelen } \\
\text { demostrarse por medio de la elaboración y defensa }\end{array}$ \\
\hline $\begin{array}{l}\text { Planificación e Innovación } \\
\text { Educativa en Educación } \\
\text { Primaria }\end{array}$ & $\begin{array}{l}\text { de su área de estudio. } \\
\text { CE4. Diseñar y regular espacios de aprendizaje en } \\
\text { contextos de diversidad y que atiendan a la igualdad } \\
\text { de género, a la equidad y al respeto a los derechos }\end{array}$ \\
\hline $\begin{array}{l}\text { Convivencia Escolar y Cultura } \\
\text { de Paz en Educación Primaria }\end{array}$ & $\begin{array}{l}\text { humanos que conformen los valores de la formación } \\
\text { ciudadana. } \\
\text { CE10. Reflexionar sobre las prácticas de aula para } \\
\text { innovar y mejorar la labor docente. Adquirir hábitos y } \\
\text { destrezas para el aprendizaje autónomo y } \\
\text { cooperativo y promoverlo entre los estudiantes. } \\
\text { CM2.11. Conocer y aprender experiencias } \\
\text { innovadoras en educación primaria. }\end{array}$ \\
\hline
\end{tabular}

Figura 1. Selección de competencias comunes de Educación Primaria.

Fuente: Elaboración propia

Para la realización de la primera actividad, se elaboraron vidrieras en grupos de trabajo cooperativo, realizadas en papel celofán de colores y cartulina negra e insertadas en un panel de corcho extruido. Cada grupo tuvo que representar de forma geométrica y sencilla un dibujo, donde escenificaron uno de los 12 Derechos Humanos, 
de los cuales 6 son Derechos Sociales y los otros 6 son Derechos Universales (véase figura 2).

\begin{tabular}{l|l}
\hline \multicolumn{1}{c|}{ Derechos Sociales } & \multicolumn{1}{|c}{ Derechos Universales } \\
\hline $\begin{array}{l}\text { Derecho a un empleo y a un } \\
\text { salario justo. }\end{array}$ & $\begin{array}{l}\text { ARTíCULOS } 1 \text { y } 2 \\
\text { Derecho a ser libres e iguales en dignidad y derechos } \\
\text { sin distinción alguna de raza, color, sexo, idioma, } \\
\text { religión, opinión política o de cualquier otra índole, } \\
\text { origen nacional o social, posición económica, } \\
\text { nacimiento o cualquier otra condición. }\end{array}$ \\
\hline $\begin{array}{l}\text { Derecho a la protección social en } \\
\text { casos de necesidad (seguridad } \\
\text { social, bajas laborales, } \\
\text { desempleo, jubilación, maternidad, } \\
\text { etc.). }\end{array}$ & $\begin{array}{l}\text { ARTíCULO 13 } \\
\text { Derecho a poder circular libremente por el mundo } \\
\text { (Migración= Inmigración y emigración). }\end{array}$ \\
\hline $\begin{array}{l}\text { Derecho a la vivienda. } \\
\text { Derecho a una educación gratuita } \\
\text { y de calidad. }\end{array}$ & $\begin{array}{l}\text { ARTíCULO } 5 \\
\text { Derecho al respeto: no agresiones a las PSH en la calle, } \\
\text { ancianos, niños, inmigrantes, racismo y xenofobia) }\end{array}$ \\
\hline $\begin{array}{l}\text { Derecho a un juicio justo y derecho a un abogado de } \\
\text { oficio. }\end{array}$ \\
\hline $\begin{array}{l}\text { Derecho a la sanidad. } \\
\text { soberanía alimentaria. }\end{array}$ & $\begin{array}{l}\text { ARTíCULO 19 } \\
\text { Derecho de libre expresión: manifestarse, escribir, etc. }\end{array}$ \\
\hline & $\begin{array}{l}\text { ARTíCULO 29 } \\
\text { Derecho a desarrollarse en comunidad con otras } \\
\text { personas y respetarlas, no estar solos. }\end{array}$ \\
\hline
\end{tabular}

Figura 2. Selección y adaptación de Derechos para la AAD de las vidrieras. Fuente: Elaboración propia.

Previamente el docente seleccionará y asignará a cada grupo un derecho.

El resultado de cada grupo se compone de tres partes (véase figura 1): derecho + dibujo vidriera + mensaje de calle, que se plasmó sobre unos soportes de corcho.

De este modo, se construyeron 14 vidrieras que serán representadas a través del lenguaje visual, con la finalidad de que sean más comprensibles para los discentes de los centros escolares de la etapa de Educación Primaria que asistirán al acto y el público en general (véase figura 3). 


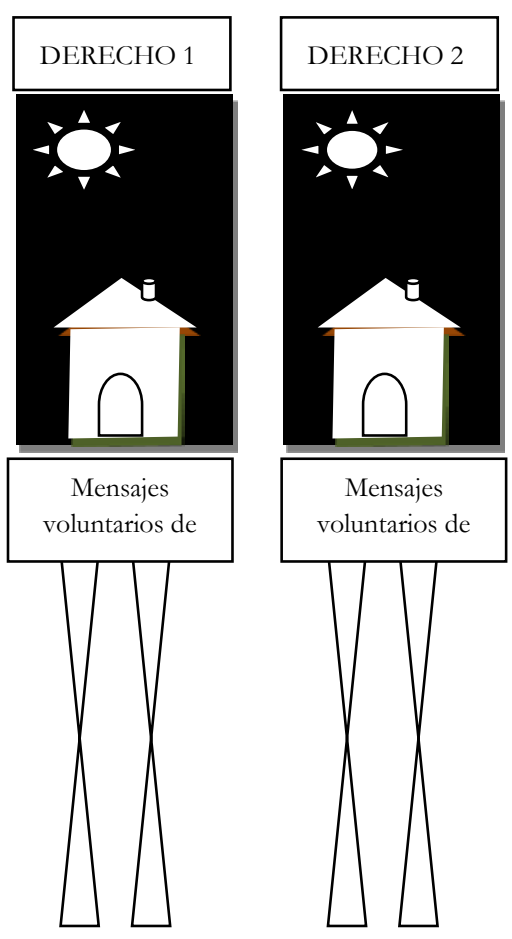

Figura 3. Descripción de la estructura de las vidrieras.

Elaboración propia.

Las medidas de la estructura exigidas al alumnado, para la realización de las vidrieras, fueron las siguientes: $22 \mathrm{~cm}$ de ancho y $2,13 \mathrm{~m}$ de altura $(20 \mathrm{~cm}$ para el papel del derecho (parte superior), $33 \mathrm{~cm}$ para la vidriera, $70 \mathrm{~cm}$ para los mensajes de la calle (mensajes voluntarios) y $90 \mathrm{~cm}$ para los soportes).

Por otro lado, se solicitó a los estudiantes que con el corcho restante realizaran todas las letras del lema, "Somos Personas, Tenemos Derechos, Nadie Sin Hogar", en los colores rojo y blanco, tonalidades de Cáritas Diocesana.

Una vez finalizadas las vidrieras, los discentes delante de su panel y vestidos de negro con una máscara blanca, tuvieron que representar con el cuerpo mediante la mímica y en silencio, el derecho asignado a su vidriera, e ir repetiéndola tantas veces como marque la secuenciación musical, puesto que dichas representaciones fueron acompañadas de varias piezas musicales, elegidas y ensayadas con el docente en el aula.

Al mismo tiempo, se realizó una actividad de empatía donde el alumnado asistió a una charla impartida por Cáritas Diocesana en la Casa de Acogida "Madre del Redentor", en la que cuatro PSH dieron su testimonio de vida, que fue reforzado con un taller sobre competencias emocionales, impartido por el profesorado implicado, al que asistieron los discentes para trabajar la conducta empática, especialmente, retomando muchos de los testimonios de dichas personas. 
La representación de las vidrieras fue el día 23 de noviembre, día internacional de las PSH, donde el alumnado participó en el acto organizado por la propia Cáritas Diocesana (véase figura 4).

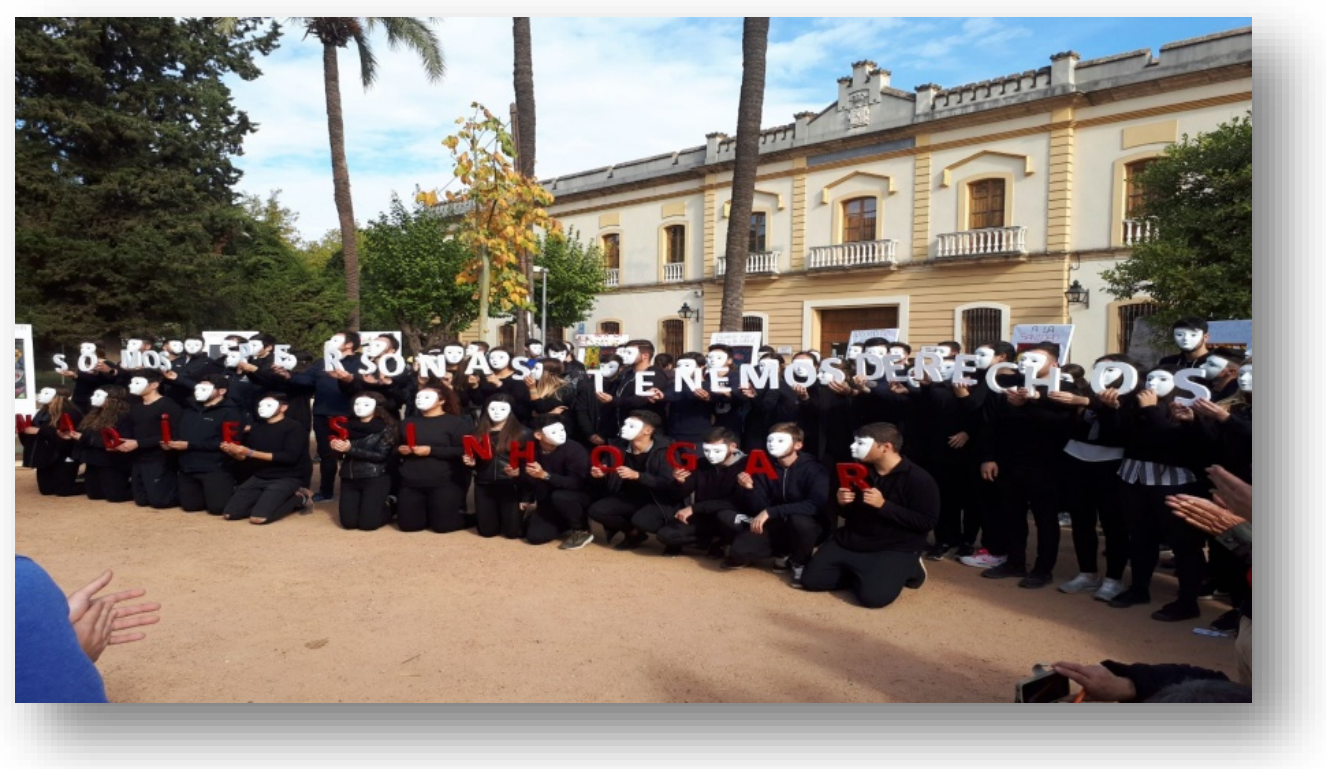

Figura 4. Representación de las Vidrieras en el Acto de sensibilización de calle. Elaboración propia.

Finalmente, el alumnado tuvo que elaborar en grupo, un documento digital en formato Word, que incluyó de forma sintetizada las siguientes partes:

$>$ Contextualización de la Casa de Acogida "Madre del Redentor" de Cáritas Diocesana: descripción, gestión, organización y funcionamiento de esta. El texto debió redactarse siguiendo la normativa APA en su última versión para citar las fuentes consultadas.

$>$ Proceso de elaboración de la vidriera: todos los grupos de trabajo siguieron los mismos pasos para la realización de las vidrieras, siendo los siguientes:

- Distribución del material (corcho, papel de celofán de colores, cúter, pegamento, etc.) y las medidas de las vidrieras, por el profesorado.

- Diseño del dibujo por los discentes, en función del derecho asignado a cada grupo.

- Realización del marco de corcho para insertar la vidriera.

- Se forra dicho marco de papel continuo blanco, para dar uniformidad al conjunto y se escribe con rotulador en la parte superior del corcho ya forrado: el derecho correspondiente, por ej.: "derecho a la libertad de expresión".

- Se dibuja en la cartulina negra, el dibujo consensuado por el grupo y se recorta dejando el contorno del dibujo.

- Se pega el papel celofán de colores en los diferentes huecos de la cartulina.

- Se inserta la vidriera en el marco preparado para esta (Véase figura 5).

Testimonios: el impacto que les ha causado haciendo una descripción y valoración de ellos. 


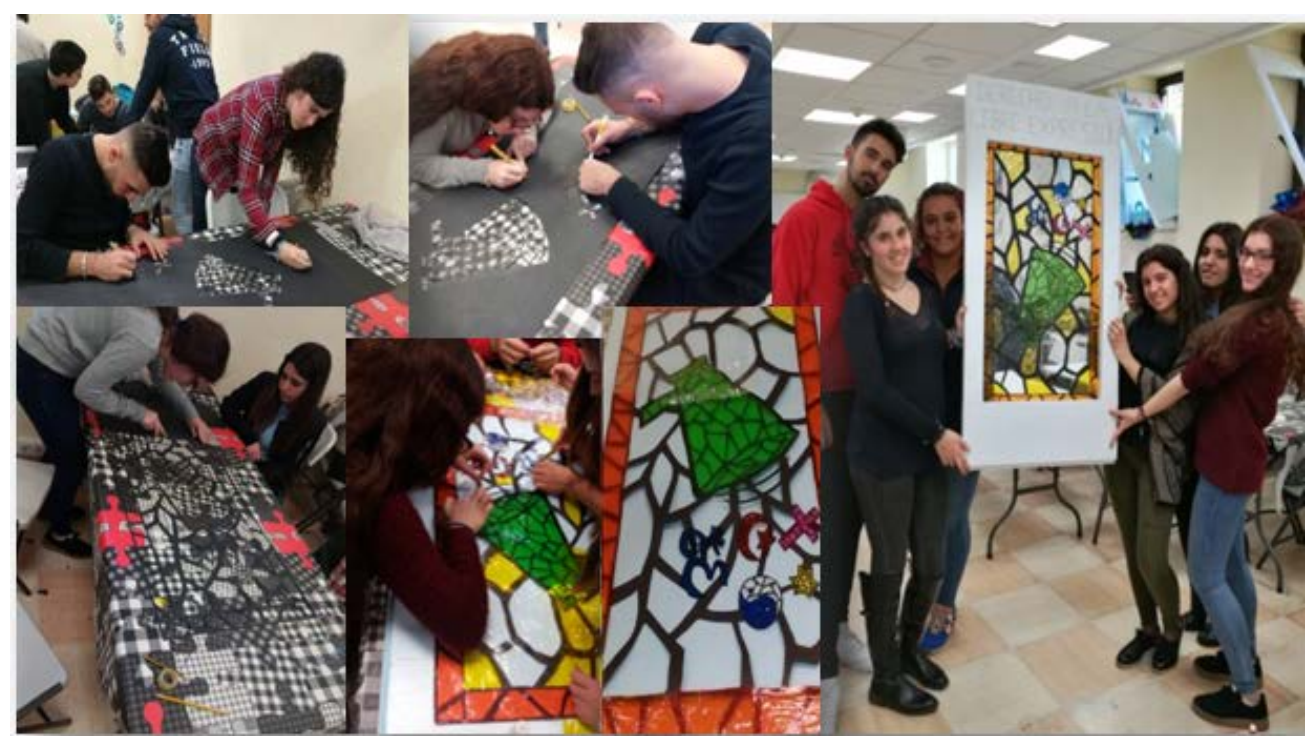

Figura 5. Proceso de elaboración de la vidriera. Elaboración propia.

En segundo lugar, con respecto al alumando de Educación Infantil, se realizó una representación teatral, en la que intervinieron las siguientes materias: Expresión Plástica Infantil y su Didáctica y Habilidades Comunicativas, Sociales, de Gestión y Emprendimiento para la Función Docente. Para ello, la selección de competencias fueron las siguientes (véase figura 6):

\begin{tabular}{l|l}
\hline \multicolumn{1}{c|}{ ASIGNATURAS } & \multicolumn{1}{c}{ COMPETENCIAS } \\
\hline $\begin{array}{l}\text { Expresión Plástica Infantil y su } \\
\text { Didáctica }\end{array}$ & $\bullet \begin{array}{l}\text { CB4. Que los estudiantes puedan transmitir } \\
\text { información, ideas, problemas y soluciones a un público } \\
\text { tanto especializado como no especializado. }\end{array}$ \\
$\begin{array}{l}\text { CE4. Fomentar la convivencia en el aula y fuera de ella } \\
\text { yabilidades Comunicativas, } \\
\text { Sociales, de Gestión y } \\
\text { Emprendimiento para la } \\
\text { Función Docente }\end{array}$ & $\begin{array}{l}\text { observar sistemáticamente contextos de aprendizajes y } \\
\text { convivencia y saber reflexionar sobre ellos. }\end{array}$ \\
& $\begin{array}{l}\text { CM11.2. Conocer y aplicar los procesos de interacción } \\
\text { y comunicación en el aula, así como dominar las }\end{array}$ \\
& clima que facilite el aprendizaje y la convivencia.
\end{tabular}

Figura 6. Selección de competencias comunes de Educación Infantil. Fuente: Elaboración propia.

La actividad se desarrolló en grupos de trabajo cooperativo y de forma conjunta entre el alumnado y algunas personas $\mathrm{PSH}$, que ejercieron de voluntarios en los grupos de trabajo de forma interactiva, para elaborar todo el material necesario y acorde con la escenificación: máscaras, marionetas, vestuario y atrezzo (Véase figura 7). 


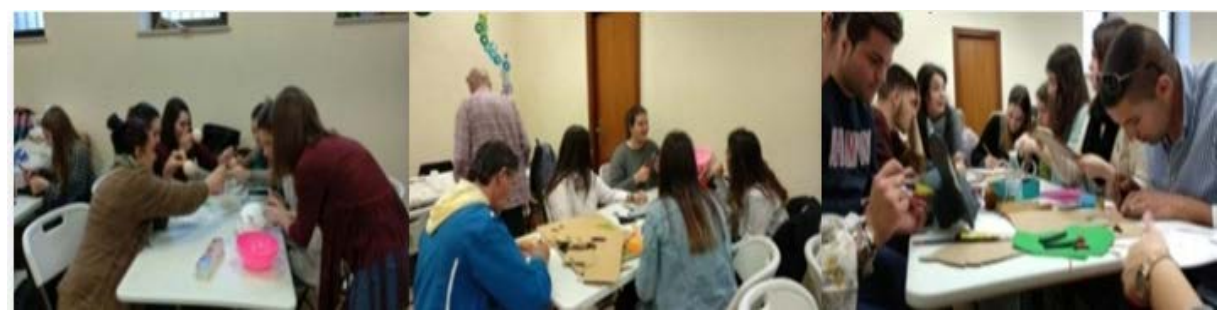

Figura 7. Grupos de trabajo cooperativo en el aula. Fuente: Elaboración propia.

Por último, el alumnado elaboró de forma individual una reflexión sobre la experiencia vivida en la actividad ejecutada.

Fase III. Evaluación. Para esta fase, se diseñaron instrumentos de evaluación que permitieron valorar el logro de competencias y los objetivos propuestos, durante y después de la experiencia llevada a cabo. En primer lugar, en la secuenciación temporal de la elaboración de las vidrieras y la preparación del material para la representación escénica y la representación teatral, se emplearon listas de control. Además para la entrega teórica de actividades programadas, se utilizaron escalas de valoración que fueron diseñadas de forma específica para cada titulación, puesto que para Educación Primaria el trabajo fue grupal, mientras que para Educación Infantil, fue individual. De dichas valoraciones y reflexiones, se han obtenido futuras propuestas de mejora.

\section{RESULTADOS}

Este proyecto de innovación educativa se centra en el aprendizaje y logro de competencias del alumnado del Centro de Magisterio "Sagrado Corazón" como futuros docentes, utilizando metodologías basadas en trabajos de grupo cooperativo.

De esta forma, la ejecución de las actividades planificadas, centradas en tareas innovadoras y gratificantes, implica la conexión y continuidad entre las materias participantes, logro que queda reflejado en la adquisición de competencias del alumnado.

Los resultados previstos son los siguientes:

- Concienciación y sensibilizacion de la realidad de las PSH, así como el conocimiento del funcionamiento de la Casa de Acogida "Madre del Redentor" de Cáritas Diocesana.

- Adquisición de valores de respeto, solidaridad y empatía por este sector de la sociedad.

- Desarrollo de competencias a través del trabajo cooperativo e interactivo.

- Fomento de la interdisciplinariedad como método de trabajo.

- Elaboración de actividades centradas en tareas innovadoras y gratificantes que impliquen la conexión y continuidad entre la teoría y la práctica, a través de AAD.

- Mejora de la formación académica y profesional del alumado participante.

Finalmente, la implementación de las actividades programadas en el proyecto, permiten optimizar el aprendizaje del alumnado empleando una perspectiva interdisciplinar, y a su vez, les hace tomar conciencia de la necesidad que estas tienen en su formación personal y profesional para el ejercicio de la función docente. 


\section{CONCLUSIONES}

Los recursos metodológicos para la adquisición de competencias y la inserción del alumnado con la realidad de las PSH, permiten una mirada conjunta de la inclusión y la invisibilidad social, empleando un enfoque participativo donde las opiniones e inquietudes de ambos colectivos se tengan en cuenta. Para ello, es preciso no poner barreras excluyentes a la educación del estudiante como futuro docente.

Asimismo, la planificación de AAD son acciones y tareas educativas con las que el alumnado puede comprender que las interacciones sociales "forman parte del proceso de la persona" (Red de Programas de Personas Sin Hogar de Cáritas, 2012, p. 263).

Por tanto, se trata de que los estudiantes universitarios se conciencien e interioricen los valores necesarios para la transferencia de aprendizajes adquiridos, a través de la experiencia vivencial con la realidad de las PSH como vehículo para el logro de competencias básicas, específicas y modulares.

Por otro lado, la mayoría de los alumnos que participaron en este proyecto, afirmaron que desconocían esta realidad social, bien por los prejuicios que tenían ante ella o por miedo, pero ya sean prejuicios, desconocimiento, miedo, timidez, vergüenza, etc., lo inquietante es que este sector de la sociedad ha aumentado considerablemente con la crisis económica de los últimos años, y que nuestro alumnado como futuros educadores, no pueden quedarse al margen. Como maestros del mañana tienen que saber cómo desenvolverse en todos los ámbitos de la sociedad, puesto que serán un modelo a seguir, y como tales, deberán ser capaces de dar respuesta a los interrogantes de sus discentes, ante dicha realidad.

Sin embargo, a pesar de dicho desconocimiento, casi la totalidad del alumnado agradeció la participación en esta experiencia a pesar de la dureza de la misma, puesto que fueron capaces de salir de la situación de confort a la que están acostumbrados, y muchos de ellos sintieron que este tipo de aprendizaje era una lección de vida, que no olvidarían jamás.

Tras este proyecto, se inició un grupo de estudiantes voluntarios que se implicaron semanalmente con esta realidad, pese a que ya han finalizado sus estudios universitarios.

\section{AGRADECIMIENTOS}

Los autores agradecen a Cáritas Diocesana especialmente a la Casa de Acogida "Madre del Redentor" y al alumnado del Centro de Magisterio "Sagrado Corazón", la ayuda y el apoyo recibido para la ejecución de este proyecto. 


\section{REFERENCIAS}

Chisaguano, J. D. (2016). Recursos didácticos lúdico-interactivos para la enseñanzaaprendizaje de las obras de arte. Quito: UCE.

Colom, A., Bernabeu, J., Domínguez, E. \& Sarramona, J. (2008).Teorías e Instituciones Contemporáneas de la Educación. Barcelona: Ariel.

Palacios, A. (2010). Educación Artística comunitaria en Finlandia. Pulso Revista de Educación, 33, 109-127. Recuperado de https://revistas.cardenalcisneros.es/index.php/PULSO/article/view/96/72

Red de programas de Personas sin hogar de Cáritas (2012). Acompañando...nos. Mirada, acción y propuestas de Cáritas con Personas que están Sin Hogar. Madrid: Cáritas Española Editores.

Rodríguez, M. (2005). Materiales y recursos en Educación Infantil. Manual de Usos prácticos para el Docente. Vigo: Editorial Ideaspropias.

Trilla, J. (coord.) (2007). El legado pedagógico del siglo XX para la escuela del siglo XXI. Barcelona: Graó. 